\title{
Delirio en pacientes con síndrome coronario agudo en una unidad de cuidados intensivos
}

\section{Incidence of delirium in patients with coronary syndrome acute in intensive care units}

\author{
Álvaro Sánchez Caraballo ${ }^{1}$ (D) Jorge Herrera Herrera ${ }^{2}$ (D) Néider Cárdenas Díaz ${ }^{3}$, \\ Edison Oyola López ${ }^{4}$
}

Recibido para publicación: Febrero 11 de 2019 - Aprobado para publicación: Mayo 28 de 2019

\begin{abstract}
RESUMEN
El Delirio es una variación aguda del estado de conciencia, frecuente en la unidad de cuidados intensivos (UCI). Su incidencia varía ampliamente, presentando diferentes características clínicas correlacionadas. Objetivo. Determinar la incidencia de delirium en pacientes con síndrome coronario agudo (SCA) en la UCl, e identificar sus características clínicas asociadas, así como la correlación del delirio (CAM-ICU) y el grado de severidad de la enfermedad (APACHE-II). Materiales y métodos. Estudio descriptivo, prospectivo, cuantitativo. Realizado durante el cuarto bimestre del 2015. A los pacientes bajo los criterios de inclusión, se les aplicó la herramienta diagnóstica CAM-ICU, para detectar la presencia de delirium, así como una revisión de la historia clínica, y operacionalización de variables. Luego de un análisis estadístico multivariado de tipo descriptivo y correlacional se obtuvieron los objetivos planteados. Resultados. La incidencia de delirio en el grupo clínico estudiado fue del $37,5 \%$, con promedio de edad para pacientes con delirium de 67 años vs 64 años para los que no presentaron. El grado de asociación entre el delirium y las características clínicas operacionalizadas presenta una significancia mayor a 0,05\%, al igual que la relación entre el delirium y el porcentaje de mortalidad según APACHE-II (Phi 283). Conclusiones. La incidencia del delirio en pacientes con SCA en la UCl fue del $37,5 \%$, evidenciando una asociación estadística no significativa entre el delirium y las características clínicas identificadas. El índice de mortalidad se comportó de forma independiente a la aparición de delirium.
\end{abstract}

Palabras clave: Delirio, incidencia, síndrome coronario agudo, unidad de cuidados intensivos.

\begin{abstract}
DELIRIUM is a variation acute state of consciousness, often in intensive care (ICU). Its incidence varies widely, featuring different correlated clinical features. Objective. To determine the incidence of delirium in patients with acute coronary syndrome (ACS) in the ICU, as well as the correlation of delirium (CAM-ICU) and the degree of severity of the disease (APACHE-II). Materials and method. Descriptive, prospective, quantitative study. Made during the fourth quarter 2015. Patients under the inclusion criteria, applied the CAM-ICU diagnostic tool, to detect the presence of delirium, as well as a review of medical history, and operationalization of variables. The objectives set were obtained after a multivariate statistical analysis descriptive and correlational. Results. The incidence of delirium in the clinical study group was $37.5 \%$, with average age for patients with delirium of 67 vs 64 years for those who did not. The degree of association between delirium and operacionalizadas clinical features have one significance greater than $0.05 \%$, as well as the relationship between delirium and the percentage of mortality according to APACHE-II (Phi 283). Conclusions. The incidence of delirium in patients with ACS in the ICU was $37.5 \%$, evidencing a non-significant statistical association between delirium and the identified clinical characteristics. The mortality rate behaved independently to the emergence of delirium.
\end{abstract}

Keywords: Delirium, incidence, acute coronary syndrome, intensive care units.

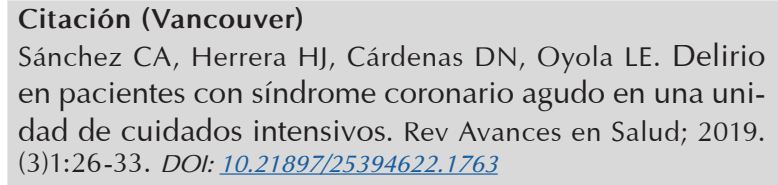

(c) 2019. Universidad de Cordoba. Este es un artículo de acceso abierto distribuido bajo los términos de la licencia Creative Commons Attribution License, que permite el uso ilimitado, distribución y reproducción en cualquier medio, siempre que el autor original y la fuente se acreditan.

1 Ph.D. en Salud Pública. Profesor Facultad Ciencias de la Salud, Universidad de Córdoba, Montería, Colombia. Correspondencia: aasanchez@correo.unicordoba.edu.co

${ }^{2}$ Esp. en Cuidados al adulto en estado crítico, coordinador de la unidad de cuidados intensivos clínica ZAYMA

3,4 Enfermero. Universidad de Córdoba, Colombia. 


\section{INTRODUCCIÓN}

El delirio según el manual diagnóstico y estadístico de los trastornos mentales $\mathrm{V}$ edición $(D S M-V)(1)$, se define como una alteración de la conciencia que se desarrolla con cambios cognitivos y perceptuales, los cuales se presentan en horas o días y un curso fluctuante en el tiempo, "que no se explica por la existencia de una demencia previa o en desarrollo" (1). Autores como Roberts et al (2), han citado acerca del papel que juega enfermería, en la evaluación de los aspectos cognitivos, ya que la detección del delirium ofrece una oportunidad de revertir las alteraciones que esta patología ocasiona, proporciona una garantía que empodera al profesional de enfermería en el cuidado individualizado, el cual reduce las consecuencias relacionadas con mortalidad, días de estancia y complicaciones.

Antes de lanzar un juicio clínico e iniciar manejo terapéutico hay que realizar un diagnóstico diferencial que permita excluir en el paciente un cuadro de demencia, para lo que según el DSM - V (1), se debe tener en cuenta que en ambas patologías se presenta una alteración cognitiva manifestada por el deterioro de la memoria, la diferencia radica en que en la "demencia el sujeto está vigil y no tiene la alteración de la conciencia característica del delirium" (1).

En Europa, investigaciones demuestran una alta incidencia de delirium en las $\mathrm{UCl}$, la cual oscila entre el $20 \%$ y $80 \%$, $(3,4)$ datos que concuerdan con investigaciones realizadas en Suramérica (5-7), estas variaciones son dependientes de las características del estudio y su población objeto (3).

De igual forma, estudios desarrollados en Sudamérica por Ceraso et al (8), reflejan que el $90,1 \%$ de los intensivistas encuestados afirman que el delirio es una entidad subdiagnosticada. En Bogotá, Colombia, Rojas et al (9), concluye porcentajes de incidencia dentro de los valores encontrados en la literatura, resaltando que oscilan entre el $11.1 \%$ y el $28,8 \%$.

El departamento de Córdoba, Colombia, no presenta antecedentes investigativos publicados sobre este tema, aun cuando cuenta con diversas instituciones de tercer nivel de atención acreditadas, y unidades de cuidados intensivos polivalentes y específicas.

Basados en lo anterior, se resalta que "en la práctica de enfermería clínica en la UCI la valoración del delirium como una alteración cognitiva es una condición compleja, común, que interfiere con la curación, recuperación y prolonga la estancia de los pacientes" (10); esto según Quiroz T et al (11), demanda un alto costo hospitalario en comparación a los pacientes hospitalizados que no presentan delirium.

\section{FISIOPATOLOGÍA}

En el estudio del delirio se han logrado avances significativos en la explicación del sus características conceptuales, epidemiología y somatización, entre otros aspectos, sin embargo, "Es poco lo que se conoce sobre la fisiopatología del delirium" (12) por lo que se han planteado diversas hipótesis, entre las que se destacan "la hipótesis de la deprivación de oxígeno, hipótesis de los neurotransmisores e hipótesis inflamatoria" (13).

\section{ETIOLOGÍA Y FACTORES DE RIESGO}

El delirio según el DSM V (1), presenta diversos factores causales los cuales se clasifican en cuatro etiologías; cuyas características en común son las alteraciones en la conciencia, cognición y percepción: el delirium debido a una enfermedad médica; delirium inducido por sustancias, que está relacionado con la intoxicación, abstinencia de sustancias o efectos secundarios de la medicación y delirio debido a múltiples etiologías, estos para ser tipificados 
como tal, se debe demostrar una relación causal directa entre el proceso patológico, el inicio y evolución del delirio. Por último se categoriza el "delirium no especificado, que debe utilizarse para el diagnóstico del delirium que no cumple los criterios para ningún tipo específico de delirium descrito" (1).

Con el fin de clasificar los factores de riesgo y explicar la predisposición y variaciones en el inicio del delirio en pacientes expuestos a factores de riesgo similares, Inouye (14), desarrolló un modelo predictivo para delirio y dividió los factores de riesgo en dos categorías: factores predisponentes, se presentan en la admisión al hospital e indican la vulnerabilidad basal, entre los que se destacan edades iguales o superiores a los 65 años, estados de demencia o deterioro cognitivo previo, depresión, ansiedad y algunas comorbilidades y enfermedades graves; y factores precipitantes, los cuales incluyen estímulos nociceptivos o lesiones y/o factores relacionados a la hospitalización que contribuyen al desarrollo de delirio resaltando fármacos entre los que se encuentran las benzodiacepinas, enfermedades neurológicas primarias y características ambientales de la $\mathrm{UCI}$, inmovilización y sondaje vesical.

Los objetivos de este estudio son determinar la incidencia del delirio en pacientes con SCA en la UCl, en el cuarto bimestre del 2015, así como identificar las características clínicas asociadas a esta enfermedad y el nivel de correlación entre el delirium (CAM-ICU) y el grado de severidad de la enfermedad (APACHE-II).

\section{MATERIALES Y MÉTODOS}

Se llevó a cabo un estudio, descriptivo, prospectivo, de cohorte longitudinal, cuantitativo, en todos los pacientes hombres o mujeres mayores de 18 años que se encuentren hospitalizados en la $\mathrm{UCl}$ con diagnóstico de SCA en el cuarto bimestre de 2015. En la muestra se incluyó todo paciente que cumpliera los siguientes criterios: escala de Glasgow igual o superior a 13/15, tiempo de entubación mayor a 24 horas y que el paciente o familiar haya firmado el consentimiento informado. Se excluyeron los pacientes sometidos a ventilación mecánica invasiva y no invasiva, trastornos psiquiátricos, algún tipo de demencia previamente diagnosticada, y aquellos que cursen con alteración cerebral tipo encefalopatía o enfermedad encefálica vascular. Obteniéndose un tamaño muestral de 24 pacientes.

El instrumento para el diagnóstico de delirio fue el Confusion Assessment Method for the intensive Care Unit (CAM-ICU) (15), el cual de acuerdo a lo planteado por Toro, AC, et al (16), presenta una sensibilidad del 79,4\%; especificidad del 97,9\%; valor predictivo positivo (VP+) del 93,1\%; y valor predictivo negativo (VP-) del 93,0\%; estudio en el cual se demostró un buen cumplimiento y excelente fiabilidad al desarrollarse a gran escala por enfermería.

La aplicación del CAM-ICU $(15,17)$, consta de dos pasos, en el primero se evalúa la sedación mediante la escala de Agitación y Sedación de Richmond (RASS), escala validada con altos índica de confiabilidad, la cual asigna un puntaje de $-5 \mathrm{a}+4$ de acuerdo al estado del paciente, iniciando en la no respuesta a estímulos verbales y dolorosos hasta el estado combativo y violento respectivamente, en el que pacientes con puntuaciones entre -4 o -5 se debe posponer la evaluación; el segundo paso evalúa el delirio bajo cuatro criterios; el primer criterio evaluación el estado mental, teniendo en cuenta si su alteración inicia en forma aguda o presenta un curso fluctuante; el segundo criterio evalúa la inatención mediante el examen auditivo para la atención desarrollado un componente auditivo y visual; el tercer criterio evalúa el pensamiento desorganizado, mediante la verbalización de cuatro preguntas a las cuales el paciente debe responder si o no; el último criterio evalúa si el paciente presenta alteración en el nivel de conciencia. 
Al identificar a los pacientes con síndrome coronario agudo que cumplieron con los criterios de inclusión y exclusión y previo consentimiento informado, se les aplicó el CAM-ICU cada veinticuatro horas hasta que el paciente presente delirio, egresó de la unidad funcional o falleció, posteriormente se realizó la revisión de la historia clínica para identificar grado de severidad de la enfermedad determinado por la escala de Acute Physiology and Chronic Health Evaluation II (APACHE II), tomando como referencia la puntuación dada al ingreso a la $\mathrm{UCl}$; de igual forma se definió como características clínicas las variables determinadas por los exámenes de laboratorio tipo química sanguínea, hemograma tipo III e ionograma, de los cuales se omitieron algunas variables por la falta de sus datos en un alto porcentaje de la muestra; al ser estos exámenes de uso frecuente en la $\mathrm{UCI}$ se tomaron como valores de análisis en los pacientes con diagnósticos de delirio, los resultados de los estudios con fecha de extracción de la muestra más cercanos al día en que se presentó esta alteración, por el contrario en los pacientes que no presentaron delirio se realizó una tabulación de todos los resultados de los exámenes que se le realizaron desde que ingresó como muestra del estudio hasta que egresó de la unidad funcional o falleció, seleccionando como valor de análisis para estudio, la moda entre los datos tabulados en cada variable.

La tabulación y filtro de los datos se realizaron en el software Microsoft Excel 2010; la identificación de la correlación entre las variables seleccionadas se realizó mediante el software estadístico SPSS, versión 22.0, empleando el coeficiente de asociación $\mathrm{V}$ de Cramer, el cual varía entre 0 y 1 , siendo 0 en caso de independencia y 1 en caso de dependencia perfecta. Esta medida de asociación acompaña su correspondiente nivel crítico (Sig. aproximada), el cual permite decidir sobre la hipótesis de independencia H_0:V=0 vs $H_{-} 1: V \neq 0$, la cual se rechaza cuando el valor de la significancia aproximada es menor que 0.05
Antes del proceso de investigación, se informó a los pacientes sobre los objetivo del estudio, incluyendo los riesgos y beneficios de su participación en él. El presente estudio se considera de riesgo mínimo en concordancia con lo planteado en la resolución 8430 de 1993, artículo 11, del Ministerio de salud.

\section{RESULTADOS}

El presente estudio se realizó en la UCI polivalente de una institución de la ciudad de Montería, Colombia, en el cuarto bimestre del presente año, en este periodo ingresaron 134 pacientes de los cuales 24 (17,9\%) cumplieron con los criterios de inclusión, de estos, 9 $(37,5 \%)$ presentaron delirio; al caracterizarlos por sexo, edad y diagnóstico clínico de ingreso, se evidencia que: el $77,8 \%$ fueron hombres $(n=7)$, y el $22,2 \%$ mujeres $(n=2)$, los pacientes con delirio presentaron un promedio de edad de 67.44 años vs 62,8 años para los que no presentaron esta alteración, con una desviación estándar de 10.47 y 12,4 respectivamente..

Los pacientes con delirio presentaron una frecuencia igual al 33,3\% en relación al grupo de edad en el que se presentó la alteración, así: de 50 a 60 años $(n=3)$, de 61 a 70 años $(n=3)$ y > de 71 años $(n=3)$, edad máxima de 86 y mínima de 53 años.

Como criterio de inclusión se analizaron pacientes diagnosticados con síndrome coronario agudo, de los cuales, aquellos que presentaron delirio se clasificaron en un $77,8 \%$ $(n=7)$ como infarto agudo de miocardio, con una frecuencia de 4 casos $(44,5 \%)$ los cuales no presentaron elevación del segmento ST; la angina se presentó en el 22,2\% ( $n=2)$ de los pacientes, clasificada en un $11,1 \%$ como angina inestable e igual proporción como angina inestable.

El grado de asociación entre el Delirium y el Apache es 0.283 (28.3\%), dato estadísticamente bajo, el valor de la significancia aproximada es 
de 0.589 en cual es mayor que 0.05

Al analizar la base de datos realizada en Excel, en la que se consolidan las características clínicas prevalentes, determinadas por los exámenes de laboratorio tipo química sanguínea, hemograma e ionograma, de los pacientes con delirio en la unidad de cuidados intensivos, no se comprobó asociación estadística representativas mediante el coeficiente $V$ de cramel y Phi entre estas variables, las cuales reflejaron una significancia aproximada que no es inferior a 0,05, lo que para este estudio indica una relación estadística igual a cero; resaltando que las variables con mayor significancia, aun cuando esta no fue suficiente para establecer una correlación fueron el potasio $(0,090)$, plaquetas $(1,62)$, hemoglobina corpuscular media $(1,62)$ y alteración leucocitaria, específicamente basófilo $(0,187)$.

\section{DISCUSIÓN}

A nivel global, investigaciones demuestran una alta incidencia de delirium en las unidades de cuidados intensivos, con variaciones dependientes de las características del estudio y su población objeto, estas fluctuaciones según Abelha, et al (6), varían ampliamente, alcanzando valores hasta de $89 \%$.

El delirium en pacientes con síndrome coronario agudo es una patología con escasos antecedentes investigativos; en la unidad de cuidados intensivos de la clínica ZAYMA, su incidencia acumulada fue del 37,5\% (0,375), este dato está dentro de los rangos evidenciados en la literatura, ubicándose por encima de los resultados obtenidos por Ramos, et al (18), quien al estudiar el delirio en una muestra con diagnóstico clínico principal y criterios de exclusión similares a los expuestos en nuestro estudio, en una unidad de cuidados coronarios, evidenció una incidencia del 11.1\%; este aumento en la incidencia del delirio que se presentó en este estudio, de acuerdo a este autor, es de esperar, ya que el riesgo de presentar delirium en la unidad de cuidados intensivos es hasta siete veces mayor en relación con una unidad de cuidados coronario; estas variaciones se debe probablemente a las características propias de nuestra muestra, entre las que se resaltan las patologías concomitantes con las que cursa los paciente, su alto promedio de edad, que los categoriza como adultos mayores, y concomitantemente con lo expuesto por Garrido NA et al (19), estos resultado pueden estar influenciados por el nivel de estrés al que se encuentran sometidos los pacientes debido al alto nivel de complejidad de la unidad de cuidados intensivos.

El delirio presentó una mayor incidencia en hombres que en mujeres con un $77,8 \%(n=7)$ y $22,2 \%(n=2)$ respectivamente; datos que no indican una mayor probabilidad de desarrollar deliro en hombres que en mujeres, ya que esta tendencia se encuentra determinada por la frecuencia de ingresos de hombres a la $\mathrm{UCI}$, la cual fue en el tiempo de estudio de un $82 \%$. Los pacientes con delirio presentaron un promedio de edad de 67,44 años, el cual supera por cinco años el promedio de edad de los pacientes que no presentaron delirio, esta diferencia no es estadísticamente significativa, sin embargo sigue la tendencia evidenciada en la literatura, resaltando lo planteado por Ramos JL,et al (18), quien refiere que a mayor edad, mayor probabilidad de presentar delirio, de igual forma, se establece como un factor predisponente para el desarrollo de esta entidad, al estar por encima de los 65 años, parámetros establecido por Young J (20).

El nivel de asociación entre el delirio y el porcentaje de mortalidad de la enfermedad determinado por el APACHE-II presenta un coeficiente de correlación de 0.283 y significancia de 0,589, es decir, la correlación entre estas dos variables no es estadísticamente significativa, , resultados que concuerdan con lo evidenciado por Henao (21) y Rojas JA (9), quienes en su tesis doctoral y trabajo 
de investigación constataron mediante un análisis estadístico multivariado, el resultado anteriormente planteado.

Investigaciones a nivel mundial analizaron relaciones de causalidad e independencia, así como características condicionantes de la incidencia del delirio en la unidad de cuidados intensivos, cuyos resultados plantean "diferencia estadística entre los pacientes que desarrollaron delirium comparados con los que no en cuanto a edad" (9), así como entre "el uso de sedación y analgesia, se documentó mayor tasa de delirium en el grupo de pacientes bajo estas intervenciones, al igual que con el uso de benzodiacepinas" (5), en esta línea de relaciones "la presencia de catéter vesical, se asociaron significativamente a la presencia de delirium" (21), sin embargo, en la revisión bibliográfica realizada para esta investigación no se evidenciaron estudios que detallaran las características clínicas prevalentes en los pacientes con delirio en la unidad de cuidados intensivos, determinado por los exámenes de laboratorio tipo química sanguínea, hemograma y coagulación, y su asociación con esta patología, por lo que este estudio, en aras de caracterizar la muestra y profundizar el conocimiento en este aspecto, se planteó esta situación como un objetivo.

$\mathrm{Al}$ analizar la base de datos realizada en Excel, en la que se consolidan las características clínicas prevalentes, determinadas por los exámenes de laboratorio tipo química sanguínea, hemograma y coagulación, de los pacientes con delirio en la unidad de cuidados intensivos, no se comprobó asociación estadística mediante el coeficiente $V$ de cramel y Phi entre estas variables, las cuales reflejaron una significancia aproximada que no es inferior a 0,05 , lo que para este estudio indica una relación estadística igual a cero; resaltando que las variables con mayor significancia, aun cuando esta no fue suficiente para establecer una relación de asociación, fueron el potasio
$(0,090)$, plaquetas $(1,62)$, hemoglobina corpuscular media $(1,62)$ y alteración leucocitaria, específicamente basófilo $(0,187)$, datos que pueden estar relacionados alteraciones de tipo encefalopatías, con posible hipoxemia, que en la literatura se plantean como factores precipitantes del delirio, .

Estos resultados revisten importancia ya que se potencializan como una oportunidad para que el personal de Enfermería incluya dentro del proceso de atención estrategias encaminadas a prevenir y diagnosticar efectivamente el delirium en los pacientes, empoderados de un cuidado individualizado, el cual con base a lo planteado por Roberts B (22), reduce las consecuencias relacionadas con mortalidad, días de estancia y complicaciones de esta patología.

\section{CONCLUSIONES}

La incidencia de delirio en pacientes con síndrome coronario agudo en la unidad de cuidados intensivos fue del $37,5 \%$. No se comprobó correlación estadística significativa entre el deliro y el índice de mortalidad que presenta un paciente con síndrome coronario agudo al ingreso a la unidad de cuidados intensivos, así como no se evidenció asociación entre el delirio y las características clínicas determinadas por los exámenes de laboratorio tipo química sanguínea, hemograma tipo III y coagulograma, sin embargo aún la evidencia no es concluyente en este último aspecto, por lo que se sugieren próximos estudios para aumentar el conocimiento al respecto

El Método de Evaluación de confusión para La Unidad de Cuidados Intensivos (CAM-ICU) es una herramienta confiable, efectiva, sencilla y de rápida aplicación en la unidad de cuidados intensivos, por lo que se debería protocolizar su aplicación en cada institución. 


\section{CONFLICTO DE INTERÉS}

Los autores declaran no tener conflicto de interés.

\section{REFERENCIAS}

1. American Psychiatric Association. Diagnostic and Statistical Manual of Mental Disorders DSM-5. ManMag 2003.

2. Roberts B, Chaboyer W. Patients' dreams and unreal experiences following intensive care unit admission. Nurs Crit Care 2004;9(4):173-180.

3. Depósito digital de documentos Universidad Autónoma de Barcelona \{sede Web\}* Incidencia de delirium en una unidad de cuidados críticos postquirúrgicos;2011 \{acceso 14 de junio de 2015\}. Martínez Gimeno L, Oller Sales B, Moret Ruiz E. Incidencia de delirium en una unidad de cuidados críticos postquirúrgicos. Disponible en http://ddd. uab.cat/record/81076.

4. Herrejón EP. Delirio en el enfermo crítico: nuevas herramientas, nuevas oportunidades. Revista Electrónica de Medicina Intensiva \{revista en Internet\}* 2004. \{acceso 14 de junio de 2015\}; 4(4). Disponible en: http://remi.uninet. edu/2004/04/REMIED51.htm

5. Revilla HV, Rodríguez ER, Luna VMT. Tercer lugar Premio «Dr. Mario Shapiro» Incidencia de delírium en la Unidad de Cuidados Intensivos del Hospital Regional de Alta Especialidad de Oaxaca. Rev Asoc Mex Med Crit y Ter Int 2013;27(4):231236.

6. Abelha F, Veiga D, Norton M, Santos C, Gaudreau J. Delirium assessment in postoperative patients: validation of the portuguese version of the Nursing Delirium Screening Scale in critical care. Rev Bras
Anestesiol 2013;63(6):450-455.

7. González J, Barros J. Delirium en pacientes de una unidad de cuidados intermedios: estudio prospectivo. Revista médica de Chile 2000;128(1):75-79.

8. Ceraso DH, Dueñas CC, Raimondi $N$, Celis E, Carrillo R, Ubiergo SU, et al. Encuesta iberoamericana sobre delirium en pacientes críticos. Medicina Intensiva 2010;34(8):495-505.

9. Rojas Gambasica JA, Valencia Moreno AA. Incidencia de delirium en las unidades de cuidados intensivos de adultos de las clínicas de la organización sanitas en Bogotá, Colombia. Rev. Médica Sanitas 15 (2): 8-13, 2012.

10. Ely EW, Shintani A, Truman B, Speroff T, Gordon SM, Harrell Jr FE, et al. Delirium as a predictor of mortality in mechanically ventilated patients in the intensive care unit. JAMA 2004;291(14):1753-1762.

11. Quiroz T, Araya E, Fuentes P. Delirium: actualización en manejo no farmacológico. Revista chilena de neuro-psiquiatría 2014;52(4):288-297.

12. Villalpando B JM. Delirium. En: D'Hyver, C y Gutiérrez-Robledo, LM. Geriatría. México: Editorial El Manual Moderno; 2006. p. 371 -.390.

13. Maldonado JR. Pathoetiological model of delirium: a comprehensive understanding of the neurobiology of delirium and an evidence-based approach to prevention and treatment. Crit Care Clin 2008;24(4):789-856.

14. Inouye SK, Viscoli CM, Horwitz RI, Hurst LD, Tinetti ME. A predictive model for delirium in hospitalized elderly medical patients based on admission characteristics. Ann Intern Med 1993;119(6):474-481. 
15. Wesley E, Truman B. The Confusion Assessement Method for the ICU (CAMICU); Training Manual: El Método para la Evaluación de la Confusión en la UCI, Manual de entrenamiento. Traducido al español por: José Luis Díaz G., Roberto A. Cruz Gervis, Juan Fernando Muñoz.

16. Toro A, Escobar L, Franco JG, Díaz GJ, Muñoz J, Molina $F$, et al. Versión en español del método para la evaluación de la confusión en cuidados intensivos, estudio piloto de validación. Medicina intensiva 2010;34(1):14-21.

17. Ely EW, Truman B, Shintani A, Thomason JW, Wheeler AP, Gordon S, et al. Supervisión del estado de sedación con el tiempo en pacientes de $\mathrm{UCl}$ : fiabilidad y validez de la Richmond AgitaciónSedación Escala (RASS). JAMA 2003; 289 (22): 2983 a 2991.

18. Ramos I, Trejo DP, Kaneo WF, Almanza MJ. Incidencia de delirium en las unidades de Cuidados Intensivos y Cuidados Coronarios del Hospital Central Militar. Revista Neurología, Neurocirugía y Psiquiatría. 2007; 40(2): Abr.-May: 41-49.
19. Garrido NA, González MÁ, García MG. Factores ambientales estresantes percibidos por los pacientes de una Unidad de Cuidados Intensivos. Enfermería intensiva 2007;18(4):159-167.

20. Young J, Inouye SK. Delirium in older people. BMJ 2007 Apr 21;334(7598):842846.

21. Henao Castaño AM. Delirium en pacientes con ventilación mecánica en la UCl: factores asociados y cuidado de enfermería \{tesis doctoral\}. Universidad Nacional de Colombia; 2013

22. Roberts B, Chaboyer W. Patients' dreams and unreal experiences following intensive care unit admission. Nurs Crit Care 2004;9(4):173-180. 